\title{
Association of Sleep Characteristics and Night Shift Work with Polycystic Ovary Syndrome: A Cross- Sectional Study
}

\section{Shuyi Shao}

Obstetrics and Gynecology Hospital of Fudan University

Huanqiang Zhao

Obstetrics and Gynecology Hospital of Fudan University

Zhiying Lu

Obstetrics and Gynecology Hospital of Fudan University

\section{Xiaohong Lei}

Obstetrics and Gynecology Hospital of Fudan University

Ying Zhang ( $\nabla$ 13818218839@163.com )

Obstetrics and Gynecology Hospital of Fudan University

\section{Research Article}

Keywords: polycystic ovary syndrome, sleep disturbance, night shift work, infertility

Posted Date: December 22nd, 2021

DOI: https://doi.org/10.21203/rs.3.rs-1183742/v1

License: (9) This work is licensed under a Creative Commons Attribution 4.0 International License. Read Full License 


\section{Abstract}

Background: The association between polycystic ovary syndrome (PCOS) and circadian rhythm has been established, but to date, investigations that describe the sleep-wake behavior of PCOS in China are limited. Here, we examined the association of sleep characteristics and night shift work with the risk of PCOS in Chinese women, and investigated their relationship to infertility in PCOS.

Methods: From March 21, 2021, to April 31, 2021, a total of 3927 Chinese women with or without PCOS were recruited online. All participants completed the WeChat-based electronic questionnaires. Sleep characteristics were measured by the Pittsburgh Sleep Quality Index.

Results: A total of 2871 women were extracted for final analysis. Sleep duration (OR, $0.857 ; 95 \% \mathrm{Cl}$, 0.763-0.963), sleep midpoint (OR, 1.142; 95\% Cl, 1.049-1.244), sleep disturbance (OR,1.320; $95 \%$ Cl, 0.9571.146), daytime dysfunction (OR, 1.136; $95 \% \mathrm{Cl}, 1.030-1.253)$ and night shift work (OR, $1.628 ; 95 \% \mathrm{Cl}$, 1.264-2.097) were associated with higher risk of PCOS. After adjusting for confounders including age, body mass index, smoking status, coffee and tea drinking status, sleep disturbance $(\mathrm{OR}, 1.314 ; 95 \% \mathrm{Cl}$, 1.111-1.555), daytime dysfunction (OR, 1.143; $95 \% \mathrm{Cl}, 1.034-1.264)$ and night shift work (OR, 1.800; $95 \%$ $\mathrm{Cl}, 1.388-2.333)$ remained associated. In addition, sleep disturbance $(\mathrm{OR}, 1.887 ; 95 \% \mathrm{Cl}, 1.400-2.542)$ and subjective sleep quality $(\mathrm{OR}, 1.299 ; 95 \% \mathrm{Cl}, 1.037-1.627)$ were related to infertility in women with PCOS, and sleep disturbance $(\mathrm{OR}, 1.750 ; 95 \% \mathrm{Cl}, 1.281-2.390)$ remained significant after adjusting for confounders.

Conclusions: These results suggest that sleep disturbance, daytime dysfunction and night shift work were significantly associated with PCOS. Screening for sleep disturbance and providing appropriate treatment could be potential strategies to manage PCOS and its long-term complications.

\section{Background}

Polycystic ovary syndrome (PCOS) is a major cause of female infertility worldwide, and it is associated with an increased risk of comorbidities, including type 2 diabetes mellitus, hypertension and psychiatric disorders(1). The etiology of this syndrome remains elusive. In addition to genetic factors, mounting evidence suggests that epigenetic and environmental influences might play important roles, bridging long-term exposures and subsequent phenotypes of PCOS.(2)

The circadian clock is an intrinsic system that is responsible for the coordination of various physiological and behavioral processes of the human body.(3) Misalignment between the system and daily rhythms such as food intake or physical activity increases the susceptibility to PCOS. $(4,5)$ Additionally, common sleep disorders in modern life may also be potential contributors. Evidence from animal studies support the notion that continuous light exposure directly promotes the metabolic and reproductive hallmarks of PCOS.(6) Constant darkness treatment has been shown to desynchronize the expression of circadian clock genes and, in turn, aggravates insulin resistance in PCOS-like rodents.(7) In a population-based study, obstructive sleep apnea functioned as a cardiovascular risk factor for PCOS, and its treatment 
could ameliorate some of the cardiovascular dysfunction in these women.(8) However, little is known about which form of sleep disorders is related to PCOS prevalence and which specific phenotypes and/or comorbidities in PCOS are particularly affected. Considering that lifestyle factors are largely influenced by race and/or ethnicity, specific research to explore sleep-wake behavior in the Chinese population is necessary. Therefore, in this article, we examined the association of sleep characteristics and night shift work with the risk of PCOS in Chinese women, and investigated their relationship to infertility in PCOS.

\section{Methods}

\section{Setting and Participants}

From March 21, 2021, to April 31, 2021, we carried out a cross-sectional study of women with or without PCOS. Data for this study were obtained online. The electronic questionnaire was issued through official WeChat accounts on World Sleep Day with an article aiming to promote sleep health. Introductory information explaining the purpose and voluntary nature of our study was given at the end of the article. People who were willing to participate in this survey completed the WeChat-based electronic questionnaires anonymously. To avoid repeated submissions, each IP address was limited to submitting a questionnaire once. All data were stored on a web server, and the results were exported as Excel files.

The age of the included women in this study ranged between 18 and 45 years. Women were asked about the diseases they had been diagnosed with. Possible responses included "polycystic ovary syndrome (PCOS)," "premature ovary insufficiency (POI)," "hyperprolactinemia (PRL)," "thyroid dysfunction," "hypothalamic or pituitary diseases," "gynecological benign or malignant tumors," and "never have been diagnosed". Respondents who responded "premature ovary insufficiency (POI)," "hyperprolactinemia (PRL)," "thyroid dysfunction," "hypothalamic or pituitary diseases," or "gynecological benign or malignant tumors" were excluded. Respondents who were pregnant, breastfeeding, or taking medication for sleep problems in the past 3 months were also excluded.

Demographic (e.g., age, sex, height, weight, smoking status, and coffee and tea drinking status) and health status information (e.g., the frequency, duration, regularity and flow volume of menstruation; acne vulgaris and hirsutism; whether pregnant or breastfeeding; and infertility) were collected, followed by completion of sleep questionnaires.

\section{Assessment of Sleep Characteristics}

The primary outcome was sleep characteristics, which were assessed by the Pittsburgh Sleep Quality Index (PSQI), the most commonly applied and validated tool for sleep measurement.(9) The PQSI consists of 19 questions in seven domains: subjective sleep quality, sleep latency, sleep duration, habitual sleep efficiency, sleep disturbance, use of sleep medication and daytime dysfunction. Among them, sleep disturbance includes waking up in the middle of the night or early morning, having to get up to use the bathroom, not breathing comfortably, coughing or snoring loudly, feeling too cold, feeling too hot, having bad dreams and having pain. Its total score ranges from 0 to 21, with higher scores indicating poorer 
sleep quality. Based on these data, the sleep midpoint was calculated as the midpoint between time falling asleep and rise time (rise time-[sleep duration/2]).

\section{Assessment of Night Shift Work}

The secondary outcome in our study was night shift work, which was obtained by the question in the questionnaire "Do you need to work the night shift? (Night shift work was defined as work executed outside normal working hours (08:00-18:00) for at least three months in a year)." Answers were given in "Yes" or "No."

\section{Other Variables}

Body mass index (BMI) was calculated using self-reported height and weight.

Acne vulgaris was defined as self-reported recurring acne on the neck, chest and upper back(10); hirsutism was defined as self-reported excessive facial and/or body terminal hairs(10); and infertility was defined by the failure to achieve a clinical pregnancy after 12 months or more of regular unprotected sexual intercourse.

According to the Chinese guidelines for abnormal uterine bleeding, the normal menstrual pattern was defined as menstruation with a normal frequency ( $\geq 21$ to $\leq 35$ days), a normal duration ( $\geq 3$ to $\leq 7$ days), a normal regularity (shortest to longest cycle variation $\leq 7$ days) and a normal flow volume $(5-80 \mathrm{ml}) .(11)$ Variations in any of these four parameters were regarded as abnormal menstruation.

\section{Statistical analyses}

Comparisons between general characteristics were made using the $\chi 2$ test for categorical variables (smoking status, coffee and tea drinking status, infertility, hirsutism, acne vulgaris and night shift work) and Student's two-tailed t test for continuous data (age, height, weight, BMI, menstrual conditions and sleep characteristics). Binary logistic regression analysis was used to test the association between PCOS and sleep characteristics, including night shift work, sleep duration, sleep midpoint, sleep latency, sleep disturbance, subjective sleep quality and daytime dysfunction. It was also used to determine the correlation between sleep characteristics, night shift work and infertility in women with PCOS. The results were then reported as odds ratios (ORs) with 95\% confidence intervals (Cls). The ORs were further adjusted considering age, BMI, smoking status, and coffee and tea drinking status. A confidence level of $\mathrm{P}<0.05$ was considered significant. All statistical analyses were conducted in the IBM ${ }^{\circledR}$ SPSS ${ }^{\circledR} 25.0$ statistical package (SPSS Inc., Chicago, IL).

\section{Ethical approval}

The Ethics Committee of the Obstetrics and Gynecology Hospital of Fudan University approved the present study (2021-68). Informed consent was obtained from all the participants. 


\section{Results}

A total of 3927 Chinese women were recruited online. Among them, 1146 (29.2\%) women were excluded (283 not in the age range, 49 had POI, 61 had PRL, 240 had thyroid dysfunction, 19 had hypothalamic or pituitary diseases, 453 had gynecological benign or malignant tumors, 56 had pregnancy or lactation, 230 took medicine to help sleep in the past 3 months and 21 with incredible figures). The remaining 2781 women were extracted for final analysis, consisting of 660 women with PCOS (23.7\%) and 2121 women without PCOS (76.3\%) (Figure 1).

Demographic and health status information are presented in Table 1. Women with PCOS were younger (28.6 years vs. 31.2 years, $P=0.000)$, had a higher BMl $\left(23.5 \mathrm{~kg} / \mathrm{m}^{2}\right.$ vs. $22.5 \mathrm{~kg} / \mathrm{m}^{2}, P=0.031$ ) and less preferred coffee $(17.0 \%$ vs. $22.9 \%, P=0.001)$ than the control women. Women with PCOS had a higher incidence of self-reported hirsutism ( $56.4 \%$ vs. $28.2 \%, P=0.000)$, acne vulgaris $(50.2 \%$ vs. $40.2 \%, P=$ $0.000)$, infertility $(39.4 \%$ vs. $15.0 \%, P=0.000)$ and abnormal menstruation $(76.2 \%$ vs. $38.9 \%, P=0.000)$. Abnormal menstruation manifested as abnormal regularity ( $33.3 \%$ vs. $14.5 \%, P=0.000)$, frequency $(63.9 \%$ vs. $21.5 \%, P=0.000)$ and flow volume $(22.4 \%$ vs. $14.9 \%, P=0.000)$.

\section{Association of sleep characteristics and night shift work with PCOS}

Sleep characteristics measured by the PQSI were significantly associated with PCOS (Table 2). Sleep duration (OR, 0.857; 95\% Cl, 0.763-0.963), sleep midpoint (OR, 1.142; 95\% Cl, 1.049-1.244), sleep disturbance (OR,1.320; 95\% Cl, 0.957-1.146) and daytime dysfunction (OR, 1.136; 95\% Cl, 1.030-1.253) were associated with PCOS. After adjusting for confounders including age, BMI, smoking status, and coffee and tea drinking status, sleep disturbance (OR, 1.314; $95 \% \mathrm{Cl}, 1.111-1.555)$ and daytime dysfunction (OR, 1.143; $95 \% \mathrm{Cl}, 1.034-1.264)$ remained associated. Night shift work was also related to PCOS (OR, 1.628; 95\% Cl, 1.264-2.097), and its associations remained (OR, 1.800; 95\% Cl, 1.3882.333) when potential mediators, including age, BMI, smoking status, and coffee and tea drinking status were included (Table 2). Additionally, the results were basically the same in an analysis of women who were excluded due to taking medication for sleep problems in the past 3 months (see Additional file Table S1), although (after adjustment for confounders) an association between sleep disturbance and PCOS did not exist (OR, 1.985; 95\% Cl, 0.964-4.086).

The detailed sleep disturbance is presented in Supporting Information Table S2. We found that coughing or snoring loudly during sleep $(P=0.000)$, feeling too cold $(P=0.007)$ and feeling too hot $(P=0.025)$ during sleep were the main problems (see Additional file Table S2).

\section{Association of sleep characteristics and night shift work with infertility in PCOS}


As presented in Table 3, sleep disturbance (OR, 1.887; 95\% Cl, 1.400-2.542) and subjective sleep quality $(\mathrm{OR}, 1.299 ; 95 \% \mathrm{Cl}, 1.627-2.037)$ were associated with infertility in PCOS. After adjusting confounders, sleep disturbance (OR, 1.750; $95 \% \mathrm{Cl}, 1.281-2.390)$ remained significant. Night shift work was not associated with infertility in PCOS.

\section{Discussion}

The principal finding of this study is that sleep disturbance (OR, $1.314 ; 95 \% \mathrm{Cl}, 1.111-1.555)$ and daytime dysfunction (OR, 1.143; 95\% Cl, 1.034-1.264) were significantly associated with PCOS. Night shift work appears to be a potential risk factor for PCOS (OR, 1.628; $95 \% \mathrm{Cl}, 1.264-2.097)$. In particular, sleep disturbance was significantly associated with infertility in women with PCOS $(\mathrm{OR}, 1.750 ; 95 \% \mathrm{Cl}, 1.281$ 2.390).

Lifestyle, including sleep health, is essential for women with PCOS and its lifetime comorbidities.(2) In our study, we compared sleep characteristics between women with or without PCOS based on the PSQI. We found that, compared to women without PCOS, women with PCOS had more sleep disturbances, and more daytime dysfunction but had no difference in sleep latency, sleep duration, sleep midpoint or subjective sleep quality. Our results were concordant with those of previous studies. Previously, a retrospective study in Taiwan involving women with $(n=5431)$ or without $(n=21724)$ PCOS reported the high occurrence of newly diagnosed sleep disorders in PCOS (HR 1.495, 95\% Cl 1.176-1.899), based on diagnoses made by psychiatrists.(12) More recently, a large community-based cohort study with researcher-made scales found that women with PCOS had more restless sleep and more severe tiredness, despite a similar duration of sleep than women without PCOS.(13) Increased difficulty falling asleep and maintaining sleep have also been reported in numerous studies, although no difference was presented in ours. $(13,14)$ Various sleep measurement methods and the credibility of self-reporting may be responsible for the discrepancy.

Notably, we found that sleep disturbance was strongly related to infertility in PCOS. While the association of disordered sleep with altered reproductive function has been demonstrated, $(15,16)$ currently, an analysis expanded our understanding and reported that infertile women with PCOS more commonly experience sleep disturbances than those with unexplained infertility.(17) Considering the reciprocal relationship between sleep and reproductive capacity, sleep improvement efforts should be integrated actively into routine clinical practice for PCOS.

To date, only two studies have evaluated the association between shift work and PCOS, and current results remain inconclusive. Our result was consistent with the recent large population-based multicenter survey that long-term exposure to rotating night shift work had a strong association with PCOS.

(18) Nevertheless, a small sample study argued that there was no significant association between shift work and the clinical or biochemical characteristics typical of PCOS.(19) Larger epidemiologic studies with more detailed definitions of shift work (specifically, information on the amount of night-work exposure including frequency, duration, and shift schedule) are needed to confirm our results. 
We acknowledge that our study has several limitations. First, while sleep-wake misalignment is highly associated with PCOS after minimizing bias, lack of potential confounders including a family history of PCOS, parity or children, and other medical conditions (e.g., depression, diabetes and hypertension) may compromise the reliability of the results. Second, social jet lag is an important characteristic of shift workers, manifested by differences in the sleep midpoint on weekdays and rest days.(19) Hence, to ensure the rigor of the research, detailed schedule data are needed. Finally, as in any cross-sectional questionnaire survey, the direction of the association cannot be determined, and information bias cannot be excluded.

\section{Conclusions}

In conclusion, our results reported that sleep disturbance and daytime dysfunction were significantly associated with PCOS. Night shift work was a potential risk factor for PCOS. These results suggested that screening for sleep disturbance and providing appropriate treatment should be recommended in the clinical management of PCOS. Additional research is needed to elucidate 1) to what degree sleep disturbance and shift work may modulate the characteristics and long-term comorbidities of PCOS and 2) to what extent sleep interventions may reverse these associations.

\section{Abbreviations}

PCOS, polycystic ovary syndrome; PQSI, Pittsburgh Sleep Quality Index; POI, premature ovary insufficiency; PRL, hyperprolactinemia; BMI, body mass index.

\section{Declarations}

Ethics approval and consent to participate: The Ethics Committee of the Obstetrics and Gynecology Hospital of Fudan University approved the present study (2021-68). Informed consent was obtained from all the participants.

Consent for publication: Not applicable.

Availability of data and materials: The data that support the findings of this study are available from the corresponding author upon reasonable request.

Competing interests: All authors declare that they have no conflicts of interest.

Funding: No funding. 
Authors contributions: $\mathrm{YZ}$ and $\mathrm{HQZ}$ contributed to the study design. SYS, $\mathrm{XHL}$ and $\mathrm{ZYL}$ contributed to the data collection. SYS contributed to the data analysis and drafting of the manuscript. All authors have read and approved the final manuscript.

Acknowledgments: The authors are grateful to the Publicity Department and the Information Technology Sector of Obstetrics and Gynecology Hospital of Fudan University for questionnaire distribution; and the women who took part in the study.

\section{References}

1. Balen AH, Morley LC, Misso M, Franks S, Legro RS, Wijeyaratne CN, et al. The management of anovulatory infertility in women with polycystic ovary syndrome: an analysis of the evidence to support the development of global WHO guidance. Human reproduction update. 2016;22(6):687708.

2. Escobar-Morreale HF. Polycystic ovary syndrome: definition, aetiology, diagnosis and treatment. Nat Rev Endocrinol. 2018;14(5):270-84.

3. Patke A, Young MW, Axelrod S. Molecular mechanisms and physiological importance of circadian rhythms. Nature reviews Molecular cell biology. 2020;21(2):67-84.

4. Zhang B, Zhou W, Shi Y, Zhang J, Cui L, Chen ZJ. Lifestyle and environmental contributions to ovulatory dysfunction in women of polycystic ovary syndrome. BMC Endocr Disord. 2020;20(1):19.

5. Woodward A, Klonizakis M, Broom D. Exercise and Polycystic Ovary Syndrome. Adv Exp Med Biol. 2020;1228:123-36.

6. Chu W, Zhai J, Xu J, Li S, Li W, Chen ZJ, et al. Continuous Light-Induced PCOS-Like Changes in Reproduction, Metabolism, and Gut Microbiota in Sprague-Dawley Rats. Frontiers in microbiology. 2019;10:3145.

7. Li S, Zhai J, Chu W, Geng X, Chen ZJ, Du Y. Altered circadian clock as a novel therapeutic target for constant darkness-induced insulin resistance and hyperandrogenism of polycystic ovary syndrome. Translational research: the journal of laboratory and clinical medicine. 2020;219:13-29.

8. Tasali E, Chapotot F, Leproult R, Whitmore H, Ehrmann DA. Treatment of obstructive sleep apnea improves cardiometabolic function in young obese women with polycystic ovary syndrome. The Journal of clinical endocrinology and metabolism. 2011;96(2):365-74.

9. Buysse DJ, Reynolds CF, 3rd, Monk TH, Berman SR, Kupfer DJ. The Pittsburgh Sleep Quality Index: a new instrument for psychiatric practice and research. Psychiatry Res. 1989;28(2):193-213.

10. Archer JS, Chang RJ. Hirsutism and acne in polycystic ovary syndrome. Best Pract Res Clin Obstet Gynaecol. 2004;18(5):737-54.

11. Gynecologic Endocrinology Subgroup CSoO, Gynecology CMA. [Chinese guideline for diagnosis and management of abnormal uterine bleeding caused by ovulatory dysfunction]. Zhonghua Fu Chan Ke Za Zhi. 2018;53(12):801-7. 
12. Hung JH, Hu LY, Tsai SJ, Yang AC, Huang MW, Chen PM, et al. Risk of psychiatric disorders following polycystic ovary syndrome: a nationwide population-based cohort study. PloS one. 2014;9(5):e97041.

13. Mo L, Mansfield DR, Joham A, Cain SW, Bennett C, Blumfield M, et al. Sleep disturbances in women with and without polycystic ovary syndrome in an Australian National Cohort. Clinical endocrinology. 2019;90(4):570-8.

14. Moran LJ, March WA, Whitrow MJ, Giles LC, Davies MJ, Moore VM. Sleep disturbances in a community-based sample of women with polycystic ovary syndrome. Human reproduction (Oxford, England). 2015;30(2):466-72.

15. Willis SK, Hatch EE, Wise LA. Sleep and female reproduction. Curr Opin Obstet Gynecol. 2019;31(4):222-7.

16. Warland J, Dorrian J, Morrison JL, O'Brien LM. Maternal sleep during pregnancy and poor fetal outcomes: A scoping review of the literature with meta-analysis. Sleep Med Rev. 2018;41:197-219.

17. Eisenberg E, Legro RS, Diamond MP, Huang H, O'Brien LM, Smith YR, et al. Sleep Habits of Women With Infertility. The Journal of clinical endocrinology and metabolism. 2021;106(11):e4414-e26.

18. Wang F, Xie N, Wu Y, Zhang Q, Zhu Y, Dai M, et al. Association between circadian rhythm disruption and polycystic ovary syndrome. Fertility and sterility. 2020.

19. Lim AJ, Huang Z, Chua SE, Kramer MS, Yong EL. Sleep Duration, Exercise, Shift Work and Polycystic Ovarian Syndrome-Related Outcomes in a Healthy Population: A Cross-Sectional Study. PloS one. 2016;11(11):e0167048.

\section{Tables}

Table 1 Demographic and health status information of women with or without PCOS. 


\begin{tabular}{|c|c|c|c|}
\hline \multirow[t]{2}{*}{ Characteristic } & $\operatorname{CON}(n=2121)$ & $P \cos (n=660)$ & \multirow[t]{2}{*}{$P$ value } \\
\hline & Mean (SD)/N (\%) & Mean (SD)/N (\%) & \\
\hline Age (years) & $31.2(6.3)$ & $28.6(5.1)$ & $0.000^{*}$ \\
\hline Height (cm) & $162.2(14.5)$ & $161.4(5.3)$ & 0.239 \\
\hline Weight (kg) & $59.0(15.7)$ & $61.3(17.8)$ & $0.026^{*}$ \\
\hline BMI $\left(\mathrm{kg} / \mathrm{m}^{2}\right)$ & $22.5(5.9)$ & $23.5(6.4)$ & $0.031^{*}$ \\
\hline Menstruation a & & & $0.000^{*}$ \\
\hline Normal & $1296(61.1 \%)$ & $157(23.8 \%)$ & \\
\hline Abnormal & $825(38.9 \%)$ & $503(76.2 \%)$ & \\
\hline The regularity of menstruation ${ }^{b}$ & & & $0.000^{*}$ \\
\hline Regular & $1814(85.5 \%)$ & $440(66.7 \%)$ & \\
\hline Irregular & $307(14.5 \%)$ & $220(33.3 \%)$ & \\
\hline The frequency of menstruation & & & $0.000^{*}$ \\
\hline$\otimes 21 d$ & $23(1.1 \%)$ & $9(1.4 \%)$ & \\
\hline$\geq 21 \mathrm{~d}$ or $\leq 35 \mathrm{~d}$ & $1664(78.5 \%)$ & $238(36.1 \%)$ & \\
\hline$\varangle 35 d$ & $434(20.5 \%)$ & $413(62.6 \%)$ & \\
\hline The duration of menstruation & & & 0.052 \\
\hline$\varangle 3 \mathrm{~d}$ & $16(0.8 \%)$ & $4(0.6 \%)$ & \\
\hline$\geq 3 \mathrm{~d}$ or $\leq 7 \mathrm{~d}$ & $1943(91.6 \%)$ & $586(88.8 \%)$ & \\
\hline$\otimes 7 \mathrm{~d}$ & $162(7.6 \%)$ & $70(10.6 \%)$ & \\
\hline The flow volume of menstruation & & & $0.000^{*}$ \\
\hline$\bigotimes 10 \mathrm{~mL}$ & $156(7.4 \%)$ & $99(15.0 \%)$ & \\
\hline$\geq 10 \mathrm{~mL}$ or $\leq 80 \mathrm{~mL}$ & $1804(85.1 \%)$ & $512(77.6 \%)$ & \\
\hline$\bigotimes 80 \mathrm{~mL}$ & $161(7.6 \%)$ & $49(7.4 \%)$ & \\
\hline Smoking & & & 0.732 \\
\hline No & 2095 (98.8\%) & $653(98.9 \%)$ & \\
\hline Yes & $26(1.2 \%)$ & $7(1.1 \%)$ & \\
\hline Coffee drinking status & & & $0.001^{*}$ \\
\hline No & $1636(77.1 \%)$ & 548 (83.0\%) & \\
\hline
\end{tabular}




\begin{tabular}{|c|c|c|c|}
\hline Yes & 485 (22.9\%) & $112(17.0 \%)$ & \\
\hline Tea drinking status & & & $0.050 *$ \\
\hline No & 1602 (75.5\%) & $523(79.2 \%)$ & \\
\hline Yes & $519(24.5 \%)$ & $137(20.8 \%)$ & \\
\hline Infertility ${ }^{c}$ & & & $0.000 *$ \\
\hline No & $1802(85.0 \%)$ & $398(60.3 \%)$ & \\
\hline Yes & $319(15.0 \%)$ & $262(39.4 \%)$ & \\
\hline Hirsutism $^{d}$ & & & $0.000 *$ \\
\hline No & $1523(71.8 \%)$ & $288(43.6 \%)$ & \\
\hline Yes & $598(28.2 \%)$ & $372(56.4 \%)$ & \\
\hline Acne vulgaris ${ }^{e}$ & & & $0.000 *$ \\
\hline No & $1268(59.8 \%)$ & $329(49.8 \%)$ & \\
\hline Yes & $853(40.2 \%)$ & 331 (50.2\%) & \\
\hline
\end{tabular}

\section{Notes:}

a The normal menstrual pattern was defined as menstruation with a normal frequency ( $\geq 21$ to $\leq 35$ days), a normal duration ( $\geq 3$ to $\leq 7$ days), a normal regularity (shortest to longest cycle variation $\leq 7$ days) and a normal flow volume $(5-80 \mathrm{~mL})$.

${ }^{b}$ The regular menstrual pattern was defined as the time between the shortest to longest cycles within 7 days.

${ }^{c}$ Infertility was defined by the failure to achieve a clinical pregnancy after 12 months or more of regular unprotected sexual intercourse.

${ }^{d}$ Hirsutism was defined as self-reported excessive facial and/or body terminal hairs.

${ }^{\mathrm{e}}$ Acne vulgaris was defined as self-reported recurring acne on the neck, chest and upper back.

Abbreviations: PCOS, polycystic ovary syndrome; BMI, body mass index 


\begin{tabular}{|c|c|c|c|c|c|c|}
\hline \multirow{2}{*}{$\begin{array}{l}\text { Sleep characteristics and } \\
\text { night shift work }^{\text {a }}\end{array}$} & \multicolumn{2}{|l|}{ CON } & \multicolumn{4}{|l|}{ PCOS } \\
\hline & $\begin{array}{l}\text { crude OR } \\
(95 \% \mathrm{Cl})\end{array}$ & $\begin{array}{l}\text { adjusted } \\
\text { OR }^{\text {b }} \\
(95 \% \mathrm{Cl})\end{array}$ & $\begin{array}{l}\text { crude } \\
\text { OR } \\
(95 \% \mathrm{Cl})\end{array}$ & $\begin{array}{l}P \\
\text { value }\end{array}$ & $\begin{array}{l}\text { adjusted } \\
\text { OR }^{\text {b }} \\
(95 \% \mathrm{Cl})\end{array}$ & $\begin{array}{l}P \\
\text { value }\end{array}$ \\
\hline \multirow[t]{2}{*}{ Night shift work } & 1 & 1 & 1.628 & $0.000 *$ & 1.800 & $0.000 *$ \\
\hline & [Reference] & [Reference] & $\begin{array}{l}(1.264- \\
2.097)\end{array}$ & & $\begin{array}{l}(1.388- \\
2.333)\end{array}$ & \\
\hline \multirow[t]{2}{*}{ Sleep duration } & 1 & 1 & 0.857 & $0.009 *$ & 0.913 & 0.138 \\
\hline & [Reference] & [Reference] & $\begin{array}{l}(0.763- \\
0.963)\end{array}$ & & $\begin{array}{l}(0.810- \\
1.029)\end{array}$ & \\
\hline \multirow[t]{2}{*}{ Sleep midpoint ${ }^{c}$} & 1 & 1 & 1.142 & $0.002^{*}$ & 1.061 & 0.198 \\
\hline & [Reference] & [Reference] & $\begin{array}{l}(1.049- \\
1.244)\end{array}$ & & $\begin{array}{l}(0.969- \\
1.162)\end{array}$ & \\
\hline \multirow[t]{2}{*}{ Sleep latency } & 1 & 1 & 1.047 & 0.317 & 1.049 & 0.314 \\
\hline & [Reference] & [Reference] & $\begin{array}{l}(0.957- \\
1.146)\end{array}$ & & $\begin{array}{l}(0.956- \\
1.151)\end{array}$ & \\
\hline \multirow[t]{2}{*}{ Sleep disturbance } & 1 & 1 & 1.320 & $0.001 *$ & 1.314 & $0.001^{*}$ \\
\hline & [Reference] & [Reference] & $\begin{array}{l}(0.957- \\
1.146)\end{array}$ & & $\begin{array}{l}(1.111- \\
1.555)\end{array}$ & \\
\hline \multirow[t]{2}{*}{ Subjective sleep quality } & 1 & 1 & 1.061 & 0.352 & 1.063 & 0.351 \\
\hline & [Reference] & [Reference] & $\begin{array}{l}(0.937- \\
1.201)\end{array}$ & & $\begin{array}{l}(0.935- \\
1.209)\end{array}$ & \\
\hline \multirow[t]{2}{*}{ Daytime dysfunction } & 1 & 1 & 1.136 & $0.011^{*}$ & 1.143 & $0.009 *$ \\
\hline & [Reference] & [Reference] & $\begin{array}{l}(1.030- \\
1.253)\end{array}$ & & $\begin{array}{l}(1.034- \\
1.264)\end{array}$ & \\
\hline
\end{tabular}

\section{Notes:}

a Sleep characteristics including sleep latency, sleep disturbance, subjective sleep quality and daytime dysfunction were measured by the PSQI.

${ }^{\mathrm{b}}$ Adjusted for age, BMI, smoking status, and coffee and tea drinking status.

c Sleep midpoint was calculated as the midpoint between time falling asleep and rise time (rise time[sleep duration/2]).

Abbreviations: PCOS, polycystic ovary syndrome; 
Table 3 Association between sleep characteristics and night shift work and infertility in women with PCOS.

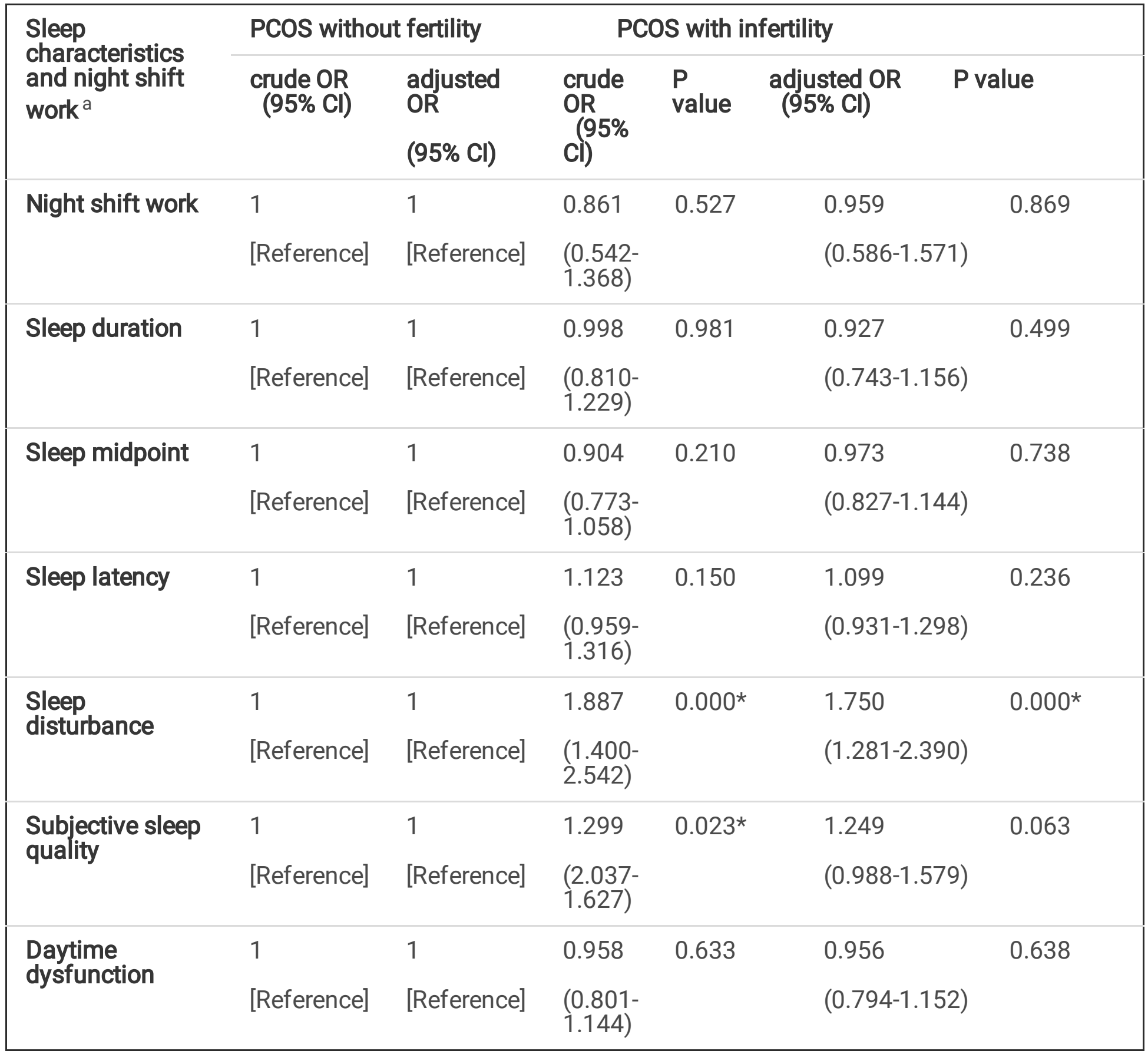

Notes:

a Sleep characteristics including sleep latency, sleep disturbance, subjective sleep quality and daytime dysfunction were measured by the PSQI.

${ }^{\mathrm{b}}$ Adjusted for age, BMI, smoking status, and coffee and tea drinking status. 
${ }^{c}$ Sleep midpoint was calculated as the midpoint between time falling asleep and rise time (rise time[sleep duration/2]).

Abbreviations: PCOS, polycystic ovary syndrome;

\section{Figures}

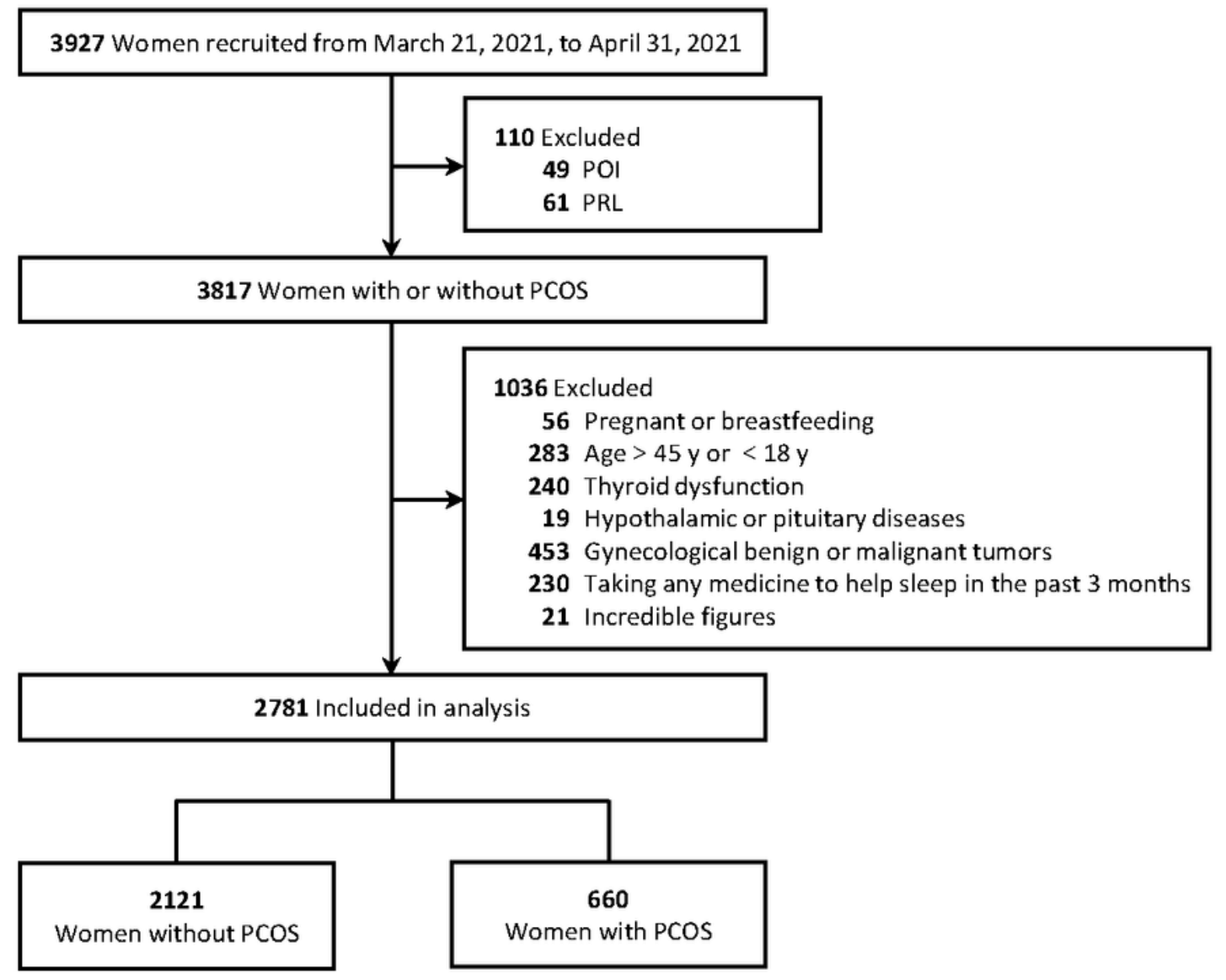

Figure 1 
Participant flow diagram.

\section{Supplementary Files}

This is a list of supplementary files associated with this preprint. Click to download.

- Additionalfile.docx 